Review article

\title{
Outcome of transplantation performed outside the regular working hours: A systematic review and meta-analysis of the literature
}

\author{
Jore Hendrikx ${ }^{a}$, Dirk Van Raemdonck ${ }^{b, c}$, Jacques Pirenne ${ }^{\text {d,e }}$, Steffen Fieuws ${ }^{\mathrm{f}}$, Steffen Rex ${ }^{\mathrm{a}, \mathrm{g}, *}$ \\ a Department of Anesthesiology, University Hospitals Leuven, Leuven, Belgium \\ b Department of Thoracic Surgery, University Hospitals Leuven, Leuven, Belgium \\ c Department of Clinical and Experimental Medicine, KU Leuven, Leuven, Belgium \\ d Department of Abdominal Transplant Surgery, University Hospitals Leuven, Leuven, Belgium \\ e Department of Microbiology and Immunology, KU Leuven, Leuven, Belgium \\ ${ }^{\mathrm{f}}$ Interuniversity Institute for Biostatistics and Statistical Bioinformatics, KU Leuven and University Hasselt, Belgium \\ ${ }^{g}$ Department of Cardiovascular Sciences, KU Leuven, Leuven, Belgium
}

\section{A R T I C L E I N F O}

\section{Keywords:}

Graft survival

Organ transplantation

Night-time

Patient survival

Weekend effect

\begin{abstract}
A B S T R A C T
Transplant procedures are frequently performed outside the regular working hours (after hours). In general surgery, several studies observed worse outcomes for operations performed after hours. The predetermined hypothesis was that patients undergoing transplantation during after hours might suffer from an excess in postoperative mortality and morbidity when compared to patients undergoing transplantations during the regular working hours. A systematic review of the PubMed database identified 11,993 records, of which eleven cohort studies including a total of 287,741 patients investigated the association between the starting time of transplant surgery and postoperative mortality (primary outcome) and/or morbidity (secondary outcome). Eight studies evaluated kidney transplants (in 165,277 patients), two studies analyzed liver transplants (in 95,346 patients), and one study investigated transplantations of thoracic organs (in 27,118 patients). Results were conflicting with two studies (in liver and lung transplantation) showing an increased mortality for transplantations performed after hours, and five studies showing no effects on mortality. A meta-analysis on estimates from four studies yielded a hazard ratio of $1.01(95 \% \mathrm{CI}, 0.98$ to 1.04$)$ for mortality comparing transplantations performed during versus outside the regular working hours. The evidence was also inconclusive for a variety of morbidity outcomes with studies demonstrating either a deterioration of outcome, no effect or an improved outcome for after hours procedures. On the basis of the available evidence, it appears impossible to give an unequivocal recommendation regarding starting times in transplant surgery.
\end{abstract}

(c) 2018 Elsevier Inc. All rights reserved.

\section{Introduction}

Transplant surgery is a distinct surgical discipline with several unique features. First, transplant patients suffer from severe and endstage disease, exposing them to a particular high risk for perioperative morbidity and mortality [1,2]. Second, transplantations are nonelective procedures with the timing of operations being unpredictable, as start times are mainly determined by the availability of donor organs and the constraints of cold ischemic time (CIT). As a consequence, transplant surgery is frequently performed at night or during the weekends (after hours). Bearing the possibly increased risks in mind when surgery is performed at night and hence by a medical team exhibiting a certain degree of sleep deprivation and fatigue [3,4], the question is obvious

\footnotetext{
* Corresponding author at: Department of Anesthesiology, University Hospitals Leuven, Herestraat 49, 3000 Leuven, Belgium.

E-mail address: steffen.rex@uzleuven.be (S. Rex).
}

whether it is safer to delay surgery until the following morning. Postponing the donation procedure would allow transplant surgery to start during regular working hours hereby attenuating the effects of fatigue. Such a strategy would however only be justified if it outweighs the potential drawbacks associated with delaying the donation and transplant procedure. These include (but are not limited to): use of intensive care unit (ICU) capacities for patients scheduled for a delayed donation procedure and hence blocking new admissions to the ICU; ignoring the wish of some families to start the donation procedure as soon as possible once the decision for organ donation is taken; and probable interference with elective operating theatre capacities/schedules in the donor hospitals [5,6]. Delaying surgery in the recipient would increase ischemia times and hence affect post-operative graft function [7].

Aim of this investigation was therefore to systematically review the available evidence for a possible association between performing transplantations outside regular working hours and surgical outcomes. The proposed hypothesis was that patients undergoing transplantation 
after hours might suffer from an excess in post-operative mortality and morbidity when compared to patients undergoing transplantations during the regular working hours.

\section{Materials and methods}

This study was conducted according to the Preferred Reporting Items for Systematic Reviews and Meta-Analyzes guidelines [8].

\subsection{Data search}

The PubMed database search ended on June 22, 2017. Different combinations of search terms were used, related to transplant surgery, time factors and outcome (see Addendum 1 for a detailed search syntax). Furthermore, a review of the references of all selected articles was performed to find other potentially relevant publications. This search strategy was set up in cooperation with experienced librarians from the Medical Library of the KU Leuven. No specific limitations were used.

\subsection{Study selection}

The selection of articles was performed in a two-step process. First a screening was realized of all the titles and abstracts of all citations to identify potentially relevant studies. Afterwards, the full text of all potentially relevant articles was reviewed to assess if they matched our inclusion criteria. In addition to this, the reference lists of all included articles were checked for any relevant articles that had not been identified in the primary search. The inclusion criteria were the comparison of outcome measurements between transplant surgery during regular working hours (daytime/weekday) and transplant procedures performed after hours (night-time/weekend). The definition of "after hours" differed among the collected studies. The term "after hours" in this article refers to weekends, evening and nights with a variation of timeframes. All observational retrospective cohort studies were included. Case reports, comments, discussion letters, publications without full text, articles in other languages than English, and conference abstracts were excluded. Eventually, all relevant studies were classified according to the transplanted organ.

\subsection{Data collection process and data items}

The data of the articles selected for this review were extracted to a database. For each study, a minimum set of data was collected; including author, journal and year of publication, country in which the study was performed, study time span, number of included patients, operative time (during the day, at night or during the weekend), main demographic characteristics and outcomes. In addition, characteristics linked to the recipient's risk were added to the database; re-transplantations, Charlson Comorbidity Score [9], CIT, time on waiting list, Elixhauser score (calculated for evaluation of non-immunologic comorbidity considering ICD-9-CM codes, [10]), model of end-stage liver disease score (MELD, [11]), donation procedure (donation after circulatory death versus donation after brain death). The data search and collection process was performed by the first author of this article.

\subsection{Outcomes}

Primary outcome of this systematic review was patient survival at any postoperative timepoint. Secondary outcomes included graft survival rates, length of hospital stay, graft function and other relevant post-operative complications.

\subsection{Statistical methods}

Only data from studies reporting hazard ratios for mortality were used in the meta-analysis. Based on a random-effects approach described by Hardy and Thompson [12], a combined estimate of the hazard ratio was obtained. Considered study estimates were loghazard ratios. The differences between estimates reflect true variability (heterogeneity) and sampling variability. Heterogeneity between studies was quantified by the $\mathrm{I}^{2}$ statistics [13], which is the percentage of total variation in study estimates that is due to heterogeneity and tested by the Cochran's $\mathrm{Chi}^{-}$square test. For two studies, there are two separate estimates. However, since there was no heterogeneity $\left(\mathrm{I}^{2}=0 \%\right)$, it was unnecessary to further distinguish between between-study and between-estimate heterogeneity. All analyses were performed using SAS software, version 9.4 of the SAS System for Windows.

\section{Results}

\subsection{Characteristics of the included studies for the systematic review}

The PubMed data search retrieved 11,993 citations. Eleven studies met our inclusion criteria (Fig. 1), all of them being observational retrospective cohort studies. Eight studies investigated kidney transplantation [5,14-20], two studies liver transplant surgery [21,22], and one study analyzed transplantations of thoracic organs [23].

Five studies described the observations of single-center investigations $[5,17,19-22]$, whereas the other six studies reported the results of a database analysis [14-16,18,21,23]. Five studies were performed in Europe: England [14,19], Austria [5], Italy [16] and the Netherlands [18]. The other six studies originate from the USA [15,17,19-23].

The outcome after transplants performed during night hours compared to during daytime was analyzed in seven studies [5,17-20,22,23], while three studies compared the patient outcome for transplant surgery in the weekend to surgery during regular working days [14-16]. One study analyzed transplant surgery performed at night-time versus daytime and during weekdays versus the weekend [21]. The definition of after hours transplant surgery varied widely among studies. In some studies only nights during weekdays were taken into account, others divided night-time into blocks of few hours. Tables 1,2 and 3 give a detailed overview of definitions used in each study. An overview of the published statistical data of the articles using hazard or odds ratio can be found in Addendum 2.

Reported outcomes included a range of parameters. Primary outcome measurements included the mortality and graft failure rates after certain time points. Secondary outcome measurements were a variation of post-operative complication rates, mainly delayed graft function, length of hospital stay (LOS), need for reoperation and incidence of surgical complications.

\subsection{Renal transplantations}

Eight studies including a total of 165,277 patients who underwent a renal transplant were identified (Table 1). 44,323 patients undergoing renal transplantation during the weekends were compared with 114,357 patients having renal transplantation during the regular weekday office hours. 2229 patients were transplanted overnight compared with 4368 patients undergoing a transplantation that started at daytime.

Four studies investigated survival rate after kidney transplantation with none of them documenting an effect for the different starting times [5,14-16]. Six studies compared graft survival with two of them reporting a significant albeit contrasting effect of starting times $[5,14,15,17,18,20]$. Fechner et al. found transplantations performed during night to increase the risk for 5-year graft failure [17]. On the contrary, Özdemir-van Brunschot et al. found a lower incidence of pure technical graft failure when renal transplant surgery was performed during the night [18]. Contrasting observations were also reported by three studies analyzing the LOS with two studies identifying a significant increase in LOS for surgeries performed during the weekend $[14,16]$, and another study, documenting a decrease in LOS for weekend procedures [15]. Likewise, the evidence was conflicting for the 


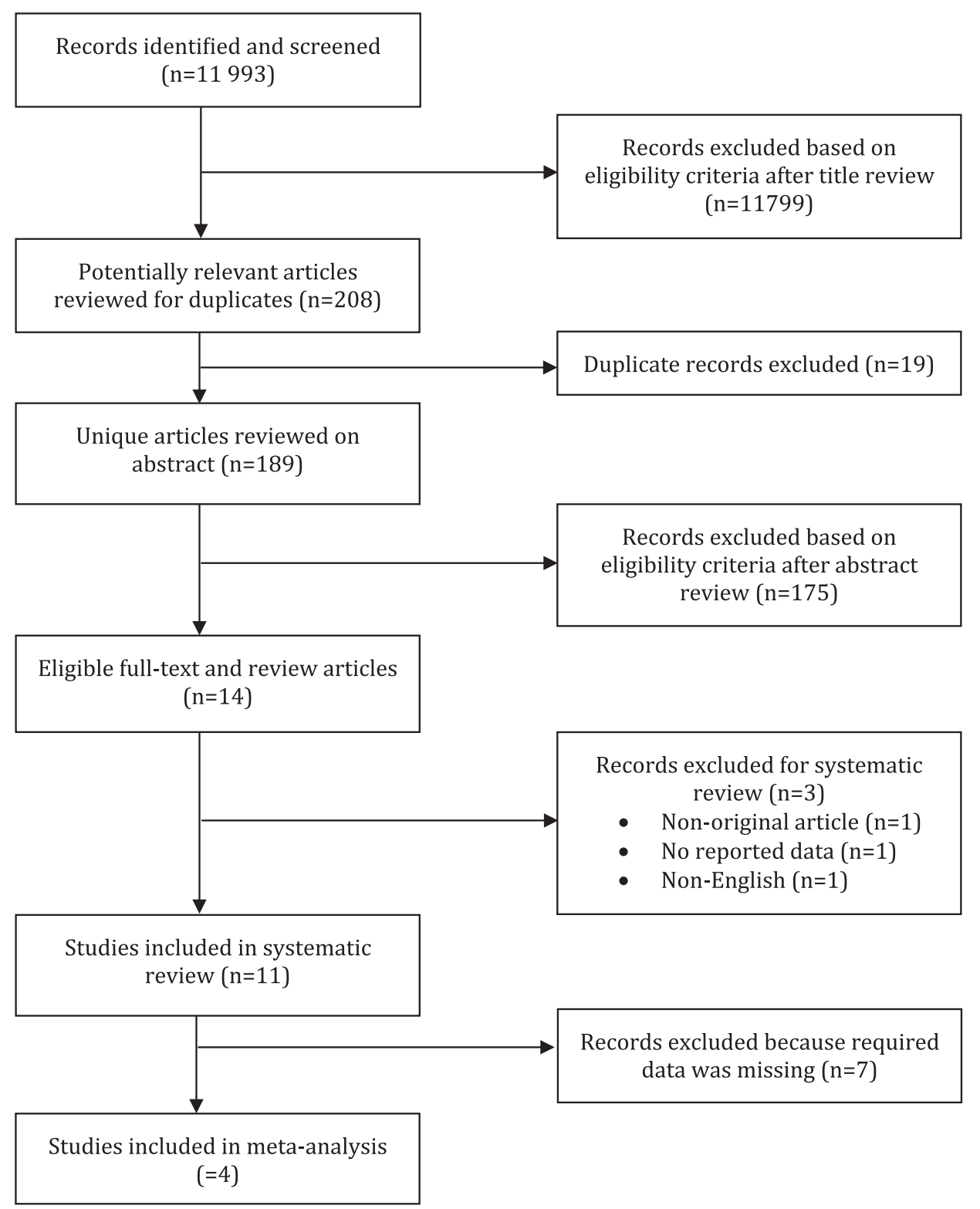

Fig. 1. Flow diagram showing the selection of studies for the systematic review and meta-analysis.

incidence of postoperative complications. One study found an increased incidence of vascular complications and re-operations within 30 days for transplant surgery performed at night [17]. This is contrasted by another study showing a lower incidence of vascular complication for nightly transplant procedures [20]. Last, one study documented a shorter operative time in kidney transplants performed during nighttime [5]. A visual analysis of the different outcome measurements for each study using hazard or odds ratios can be found as forest plots (Addendum 2A).

\subsection{Liver transplantation}

Two studies including a total of 95,346 patients who received a liver transplant were evaluated (Table 2). A total of 32,079 patients were analyzed for weekend transplant surgery and compared with 59,580 patients that underwent weekday transplant surgery. 31,333 patients underwent a liver transplantation overnight compared with 51,931 patients being operated during daytime hours.

One of the two studies found the risk of early death to be doubled and the operative time significantly increased when the liver was transplanted overnight [20]. Orman and colleagues did not observe any effects on mortality, but documented a statistically significant decrease in 1-year graft survival for the weekend compared to the weekday group [21]. A visual analysis of the different outcome measurements for each study using hazard or odds ratios can be found as forest plots (Addendum 2B).

\subsection{Thoracic organ transplantation}

Only one study analyzed the impact of starting times on outcomes after transplantations of thoracic organs, including 27,118 patients undergoing heart or lung transplantation in the United States [23]. An overview is given in Table 3. While no differences in outcome could be found for heart transplantations, 90 days mortality was reduced in patients receiving a lung transplant at night (with however no differences in 30-day and 1-year survival [21]). A visual analysis of the different outcome measurements this study using hazard ratios can be found as forest plots (Addendum 2C). 
Table 1

\begin{tabular}{|c|c|c|c|c|c|c|c|c|}
\hline Author, country, date & Study group & $\begin{array}{l}\text { Baseline donor and recipient } \\
\text { characteristics: regular hours vs. } \\
\text { after hours ( } p \text { value) }\end{array}$ & Outcome factors & Regular hours & After hours & $p$ value $^{a}$ & Ratio & Comments \\
\hline $\begin{array}{l}\text { Anderson et al. [14], } \\
\text { England } 2016\end{array}$ & $\begin{array}{l}\text { January 2003-December } 2014 \\
(n=12,902) \\
\text { Weekday: Monday to Thursday } \\
(n=7724,60 \%) \\
\text { Weekend: Friday to Sunday } \\
(n=5178,40 \%)\end{array}$ & $\begin{array}{l}\text { Donor type (\%): } \\
\text { DCD: } 25.8-24.2,(0.12) \\
\text { DBD: } 20.9-21.3,(0.12) \\
\text { Charlson Comorbidity Score, } \\
(0.18)\end{array}$ & $\begin{array}{l}30 \text { day-mortality (\%) } \\
1 \text { year-mortality (\%) } \\
\text { Allograft failure/rejection the } \\
\text { first year (\%) } \\
\text { DGF }(\%) \\
\text { LOS (days) } \\
\text { Rehospitalization }<1 \text { year (\%) }\end{array}$ & $\begin{array}{l}1.2 \\
3.8 \\
16.8 \\
\\
29.36 \\
10(7-14) \\
63.5\end{array}$ & $\begin{array}{l}0.9 \\
3.7 \\
16.7 \\
\\
29.97 \\
10(7-15) \\
63.3\end{array}$ & $\begin{array}{l}0.13 \\
0.79 \\
0.90 \\
\\
0.46 \\
<0.01 \\
0.77\end{array}$ & $\begin{array}{l}1.01(0.84-1.21)^{\mathrm{b}} \\
1.01(0.84-1.21)^{\mathrm{b}} \\
1.00(0.91-1.09)^{\mathrm{b}} \\
0.99(0.92-1.07)^{\mathrm{b}}\end{array}$ & $\begin{array}{l}\text { No inferior short-term outcome } \\
\text { on 1-year risk for } \\
\text { rehospitalization, mortality and } \\
\text { failure/rejection for renal } \\
\text { transplants performed on } \\
\text { weekdays. Length of hospital stay } \\
\text { was significantly longer for } \\
\text { weekend surgery. }\end{array}$ \\
\hline $\begin{array}{l}\text { Baid-Agrawal et al. } \\
\text { [15], USA } 2016\end{array}$ & $\begin{array}{l}\text { April } 1994-\text { September } 2010 \\
(n=136,715) \\
\text { Weekday: Monday to Friday } \\
(n=99,061,72 \%) \\
\text { Weekend: Saturday or Sunday } \\
(n=37,654,28 \%)\end{array}$ & $\begin{array}{l}\text { Donor type: DCD } \\
\text { CIT (hours): } 19(13-24) \text { vs. } 18 \\
(13-24),(<0.01) \\
\text { Retransplantations (\%): } 10.6 \text { vs. } \\
10.3,(0.12) \\
\text { Days on waiting list: } 559 \\
(228-1069) \text { vs. } 565 \text { (229-1067), } \\
(0.28)\end{array}$ & $\begin{array}{l}\text { Patient survival (years) } \\
\text { Death-censored allograft } \\
\text { survival (years) } \\
\text { Overall allograft survival } \\
\text { (years) } \\
\text { DGF (\%) } \\
\text { LOS (days) } \\
\text { Acute rejection <1 year (\%) }\end{array}$ & $\begin{array}{l}13.5(13.3-13.7) \\
12.7(12.5-12.9) \\
8.28(8.18-8.37) \\
24.9 \\
7(5-9) \\
12.7\end{array}$ & $\begin{array}{l}13.7(13.3-14.0) \\
12.8(12.5-13.1) \\
8.24(8.10-8.38) \\
24.6 \\
6(5-10) \\
12.7\end{array}$ & $\begin{array}{l}0.43 \\
0.49 \\
\\
0.29 \\
\\
0.7 \\
<0.01 \\
0.07\end{array}$ & $\begin{array}{l}1.01(0.92-1.04)^{\mathrm{b}} \\
1.01(0.99-1.03)^{\mathrm{b}} \\
1.01(0.98-1.04)^{\mathrm{b}}\end{array}$ & $\begin{array}{l}\text { The day of surgery does not affect } \\
\text { the outcome for deceased donor } \\
\text { kidney transplantations. A } \\
\text { decrease in length of hospital stay } \\
\text { was the only significant } \\
\text { association ( } 6 \text { days vs. } 7 \text { days) } \\
\text { between weekend versus } \\
\text { weekday transplant surgery. }\end{array}$ \\
\hline $\begin{array}{l}\text { Manfredini et al. [16], } \\
\text { Italy } 2016\end{array}$ & $\begin{array}{l}\text { January 2000-December } 2013 \\
(n=9063) \\
\text { Weekday: Monday to Friday } \\
(n=7572,84 \%) \text { Weekend: } \\
\text { Friday } 12 \text { p.m. to Sunday } 12 \text { p.m. } \\
(n=1491,16 \%)\end{array}$ & $\begin{array}{l}\text { Donor type: unknown } \\
\text { Elixhauser score: } 5 \text { ( }-7 \text { tot } 31) \\
\text { vs. } 5 \text { ( }-7 \text { to } 27),(n s)\end{array}$ & $\begin{array}{l}\text { In-hospital mortality and } \\
\text { cardiovascular events (\%) } \\
\text { LOS (days) }\end{array}$ & $9.5 \pm 12.3$ & $10.5 \pm 10.8$ & $\begin{array}{l}\mathrm{ns} \\
<0.01\end{array}$ & $\begin{array}{l}1.02(0.88-1.18)^{\mathrm{c}} \\
1.59(1.39-1.83)^{\mathrm{c}}\end{array}$ & $\begin{array}{l}\text { Renal transplant recipients are not } \\
\text { exposed to higher risk of adverse } \\
\text { outcome during weekend } \\
\text { transplantations. Only duration of } \\
\text { hospitalization was increased. }\end{array}$ \\
\hline $\begin{array}{l}\text { Fechner et al. [17], } \\
\text { USA } 2008\end{array}$ & $\begin{array}{l}\text { 1994-2004 } \\
(n=260) \\
\text { Day: } 8 \text { a.m.- }-8 \text { p.m. } \\
(n=166,64 \%) \\
\text { Night: } 8 \text { p.m.-8 a.m. } \\
(n=94,36 \%)\end{array}$ & $\begin{array}{l}\text { Donor type: unknown } \\
\text { CIT (hours): } 14 \pm 4 \text { vs } 14 \pm 6 \text {, } \\
\text { (ns) }\end{array}$ & $\begin{array}{l}\text { 1-year graft failure (\%) } \\
\text { 5-year graft failure (\%) } \\
\text { Reoperation <30 days (\%) } \\
\text { Ureteral (\%) } \\
\text { Vascular (\%) } \\
\text { Thrombosis graft vein (\%) } \\
\text { Hematoma (\%) }\end{array}$ & $\begin{array}{l}6.6 \\
8.4 \\
6.4 \\
1.6 \\
1.6 \\
0 \\
1.6\end{array}$ & $\begin{array}{l}10.6 \\
20.2 \\
16.8 \\
2.1 \\
8.5 \\
1 \\
4.2\end{array}$ & $\begin{array}{l}<0.05 \\
<0.01 \\
n s \\
<0.01 \\
n s \\
n s \\
n s\end{array}$ & & $\begin{array}{l}\text { Transplant surgery performed at } \\
\text { night enhances the risk for graft } \\
\text { failure, reoperation and vascular } \\
\text { complication ratio. }\end{array}$ \\
\hline
\end{tabular}


Table 1 (continued)

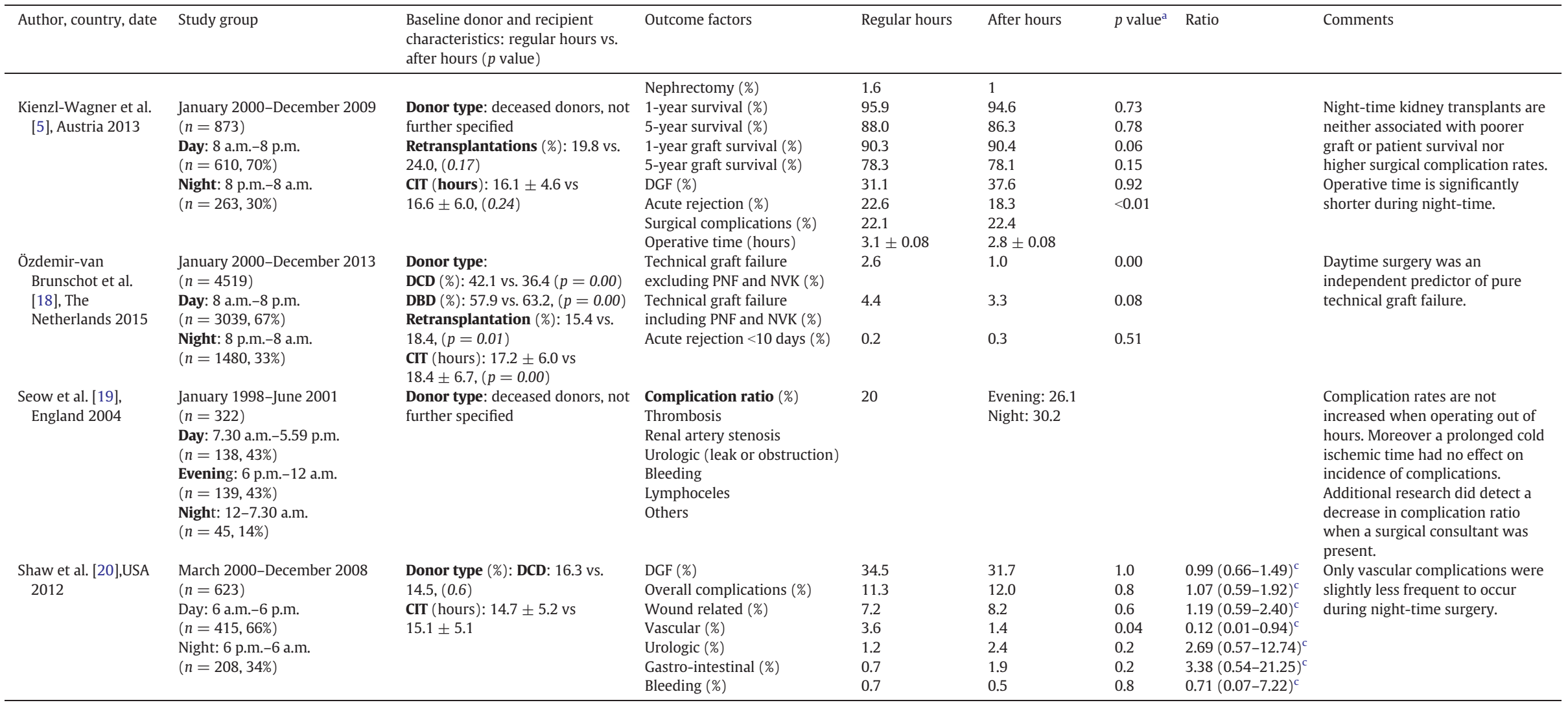

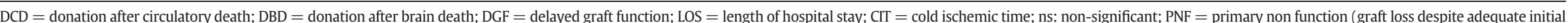
perfusion, NVK = nonviable kidneys (never-functioning renal allografts with poor perfusion); ns = not significant.

all authors considered two-tailed $p$ values $<0.05$ significant.

Adjusted hazard ratio with a $95 \%$ confidence interva.

Adjusted odds ratio with a 95\% confidence interval. 
Table 2

\begin{tabular}{|c|c|c|c|c|c|c|c|c|}
\hline $\begin{array}{l}\text { Author, country, } \\
\text { date }\end{array}$ & Study group & $\begin{array}{l}\text { Baseline donor and recipient } \\
\text { characteristics: regular hours vs. after } \\
\text { hours ( } p \text { value) }\end{array}$ & Outcome measurements & $\begin{array}{l}\text { Regular } \\
\text { hours }\end{array}$ & After hours & $p$ value $^{\mathrm{a}}$ & Ratio & Comments \\
\hline $\begin{array}{l}\text { Orman et al. [21], } \\
\text { USA } 2012\end{array}$ & $\begin{array}{l}\text { October } 1987 \text { - December } 2010 \\
(n=94,768) \\
\text { Day: } 7 \text { a.m. }-7 \text { p.m. } \\
(n=51,543,62 \%) \\
\text { Night: } 7 \text { a.m. }-7 \text { a.m. } \\
(n=31,143,38 \%) \\
\text { Weekday: Monday } 8 \text { a.m. - Friday } 5 \text { p.m. } \\
(n=59,580,65 \%) \\
\text { Weekend: Friday } 5 \text { p.m. - Monday } 8 \text { a.m. } \\
(n=32,079,35 \%)\end{array}$ & $\begin{array}{l}\text { Donor type (\%): all donors: } 82 \text { deceased } \\
\text { and } 3 \text { DCD } \\
\text { MELD era (\%): } 50 \text { vs. } 60,(0.01) \\
\text { MELD score: } 21.2 \pm 10 \text { vs } 21.3 \pm 9 \\
\text { (0.01) } \\
\text { Retransplantation (\%): } 10 \text { vs. } 10,(0.09) \\
\text { CIT (hours): } 8(6-10.5) \text { vs. } 7(5.2-9.4) \text {, } \\
(0.01)\end{array}$ & $\begin{array}{l}\text { 30-day mortality (\%) } \\
90 \text {-day mortality (\%) } \\
\text { 1-year mortality (\%) } \\
30 \text { day graft failure (\%) } \\
90 \text { day graft failure (\%) } \\
\text { 1-year graft failure (\%) } \\
\\
\text { 30-day mortality (\%) } \\
\text { 90-day mortality (\%) } \\
\text { 1-year mortality (\%) } \\
\text { 30 day graft failure (\%) } \\
90 \text { day graft failure (\%) } \\
\text { 1-year graft failure (\%) }\end{array}$ & $\begin{array}{l}\text { Day } \\
4 \\
8 \\
14 \\
8 \\
12 \\
19 \\
\text { Weekday } \\
5 \\
8 \\
14 \\
8 \\
12 \\
19\end{array}$ & $\begin{array}{l}\text { Night } \\
4 \\
7 \\
14 \\
7 \\
11 \\
19 \\
\text { Weekend } \\
5 \\
8 \\
14 \\
8 \\
12 \\
20\end{array}$ & $\begin{array}{l}0.26 \\
0.21\end{array}$ & $\begin{array}{l}0.94(0.85-1.05)^{\mathrm{b}} \\
0.98(0.90-1.06)^{\mathrm{b}} \\
0.99(0.94-1.05)^{\mathrm{b}} \\
1.00(0.92-1.08)^{\mathrm{b}} \\
1.00(0.94-1.06)^{\mathrm{b}} \\
1.01(0.96-1.06)^{\mathrm{b}} \\
\\
0.93(0.84-1.04)^{\mathrm{b}} \\
0.98(0.91-1.06)^{\mathrm{b}} \\
1.02(0.97-1.08)^{\mathrm{b}} \\
1.04(0.96-1.13)^{\mathrm{b}} \\
1.04(0.97-1.10)^{\mathrm{b}} \\
1.05(1.01-1.11)^{\mathrm{b}}\end{array}$ & $\begin{array}{l}\text { Night-time and weekend operations for } \\
\text { liver transplantations did not affect } \\
\text { graft or patient survival. There is a } \\
\text { statistically significant decline in graft } \\
\text { survival } 365 \text { days post-transplantation } \\
\text { for the weekend group. }\end{array}$ \\
\hline $\begin{array}{l}\text { Lonze et al. [22], } \\
\text { USA } 2010\end{array}$ & $\begin{array}{l}\text { June } 1995 \text { - October } 2008 \\
(n=578) \\
\text { Day: } 3 \text { a.m. }-3 \text { p.m. } \\
(n=388,67 \%) \\
\text { Night: } 3 \text { p.m. }-3 \text { a.m. } \\
(n=190,33 \%)\end{array}$ & $\begin{array}{l}\text { Donor type: DCD } 6.1 \% \text { of all donors } \\
\text { MELD score: } 20.4 \pm 8.4 \text { vs } 21.0 \pm 8.1 \text {, } \\
\text { (0.4) } \\
\text { Retransplantation (\%): } 6.4 \text { vs. } 1.6,(0.01) \\
\text { CIT (hours): } 9.3 \pm 2.3 \text { vs } 9.1 \pm 2.2,(0.4)\end{array}$ & $\begin{array}{l}\text { Early deaths }<1 \text { week (\%) } \\
5 \text {-year survival } \\
\text { Overall complications (\%) } \\
\text { Wound complications (\%) } \\
\text { Vascular complications (\%) } \\
\text { Biliary complications (\%) } \\
\text { Other complications (\%) } \\
\text { Sepsis (\%) } \\
\text { PNF (\%) }\end{array}$ & $\begin{array}{l}2.8 \\
34.3 \\
12.1 \\
9.5 \\
16.2 \\
10.6 \\
5.4 \\
2.8\end{array}$ & $\begin{array}{l}6.3 \\
40.5 \\
13.7 \\
14.2 \\
16.3 \\
9.5 \\
7.9 \\
1.6\end{array}$ & $\begin{array}{l}0.02 \\
0.5 \\
0.1 \\
0.4 \\
0.1 \\
0.9 \\
0.6 \\
0.3 \\
0.6\end{array}$ & $\begin{array}{l}2.85(1.16-7.00)^{\mathrm{c}} \\
1.34(0.93-1.94)^{\mathrm{c}} \\
1.24(0.72-2.15)^{\mathrm{c}} \\
1.56(0.90-2.68)^{\mathrm{c}} \\
1.03(0.64-1.67)^{\mathrm{c}} \\
0.84(0.46-1.54)^{\mathrm{c}} \\
1.55(0.74-3.25)^{\mathrm{c}} \\
1.50(0.33-6.86)^{\mathrm{c}}\end{array}$ & $\begin{array}{l}\text { Complications were not significantly } \\
\text { different but overnight liver transplant } \\
\text { operations were longer in duration and } \\
\text { were associated with a twofold greater } \\
\text { risk of early death compared to } \\
\text { daytime transplants. Long-term } \\
\text { survival did not differ between the } \\
\text { subgroups. Daytime surgery was } \\
\text { significantly shorter. }\end{array}$ \\
\hline
\end{tabular}

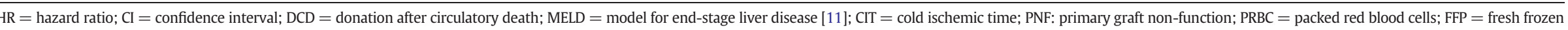
plasma.

All authors considered a two-tailed $p$ value $<0.05$ significant

Adjusted hazard ratio with a 95\% confidence interval.

Adjusted odds ratio with a 95\% confidence interval. 


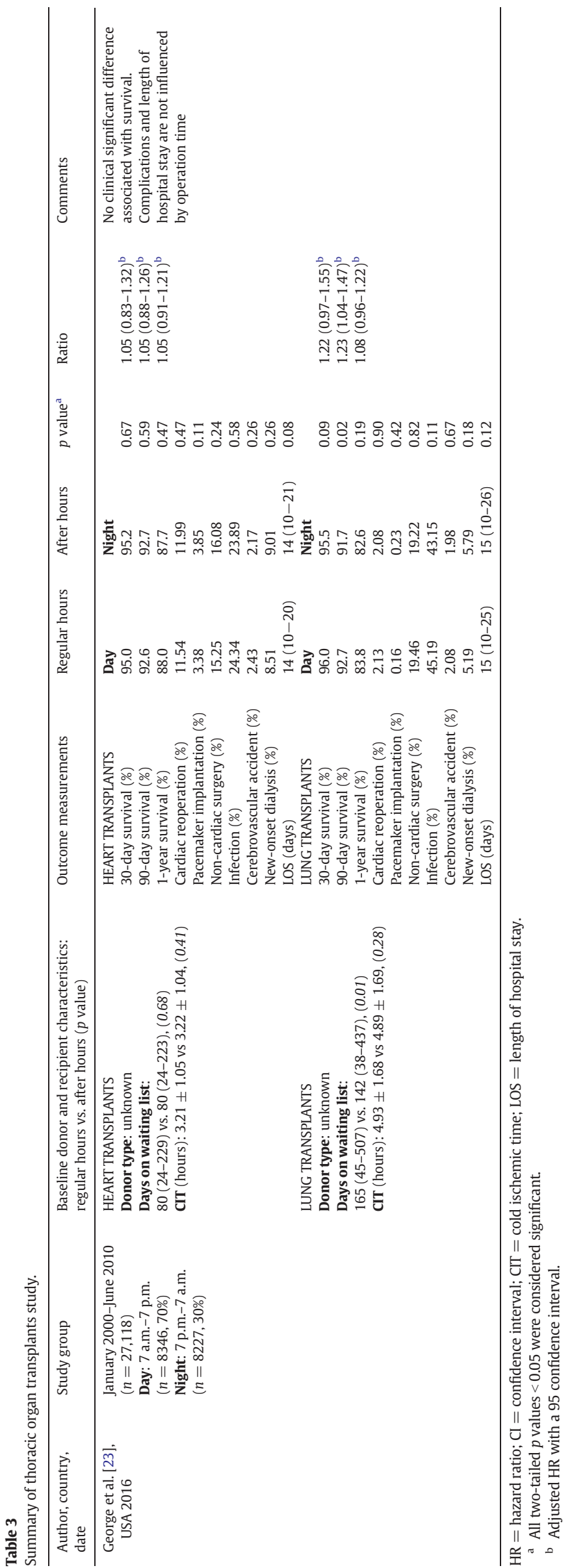

\subsection{Meta-analysis}

Only four of eleven studies reported hazard ratios from a survival analysis and could hence be integrated in a meta-analysis (Fig. 2). Two of the included studies [21,23] published two separate estimates for this outcome. In one of these studies [21], the authors investigated two different definitions for after hours: daytime versus nighttime and weekday versus weekend [21]. The other study reviewed the primary outcome for transplantations of two different thoracic organs: heart and lung [23]. In this meta-analysis, the combined hazard ratio was 1.01 ( $95 \% \mathrm{CI}, 0.98$ to 1.04 ) with a hazard ratio > 1 favoring after hours.

\section{Discussion}

For the systematic review, eleven studies were identified including 287,741 patients that analyzed the association between starting times in transplant surgery and postoperative outcome. The meta-analysis consisted of four studies, including 271,503 patients. The findings reported by the different studies were highly inconsistent with two studies showing an excess in mortality for transplantations performed after hours, and five studies and the meta-analysis showing no effects on mortality. Evidence was also inconclusive for a variety of indicators describing postoperative morbidity with studies demonstrating either a deterioration of outcome, no effect or even an improved outcome for after hours procedures.

To the best of our knowledge, this is the first systematic analysis and meta-analysis of the available evidence comparing the outcome of transplant surgery performed during regular hours (weekdays and daytime) with the outcome of procedures starting after hours (during weekends and night-time). Notably, our study encompassed kidney, liver, heart and lung transplants with the results being in line with a systematic review that exclusively analyzed outcomes of kidney transplantation, and has been published only recently and after the start of our own literature search [24].

Unfortunately, given the ambiguousness of the published evidence, it is impossible to confirm or reject our hypothesis that transplanting patients during after hours may be associated with adverse outcomes.

Interestingly, data are also surprisingly inconsistent with respect to after hours surgery in the general surgical population. In a recent secondary analysis of the European Surgical Outcomes Study database including 11,290 patients, no relationship could be observed between in-hospital mortality and time of day at which the procedure was performed when adjusting for risk factors [25]. However, in this study, several procedures known to carry a high risk were excluded from the analysis, including neurosurgery and cardiac surgery. Likewise, an analysis of 32,001 elective general surgical patients at the Cleveland Clinic could not find an association between timing of surgery and outcomes. Unfortunately, this study excluded weekend and overnight cases [26]. In 3534 adult patients undergoing elective cardiac surgery, a start time later than $4 \mathrm{pm}$ was not associated with an increase in mortality and morbidity [25].

These reports are contrasted by a variety of other studies. A retrospective analysis of 144,740 non-emergent general and vascular surgical procedures performed within the Veteran Affairs Medical System and entered into the National Surgical Quality Improvement Program Database found a significant association between starting off-hours and postoperative morbidity [28]. Accordingly, a retrospective analysis of the US National Anesthesia Clinical Outcomes Registry demonstrated that a case start time after 04:00 p.m. was associated with an excess in perioperative mortality [adjusted odds ratio of 1.64 (95\% CI, 1.22 to 2.21) for perioperative death, when comparing to starting times between 07:00 a.m. and 03:59 p.m.] [29]. Likewise, in a population of nearly 3400 cardiac surgical patients, an incision time later than 3 p.m. was associated with a more than two times higher absolute and riskadjusted mortality [30]. Similar observations were reported for percutaneous coronary interventions, with off-hours procedures being 


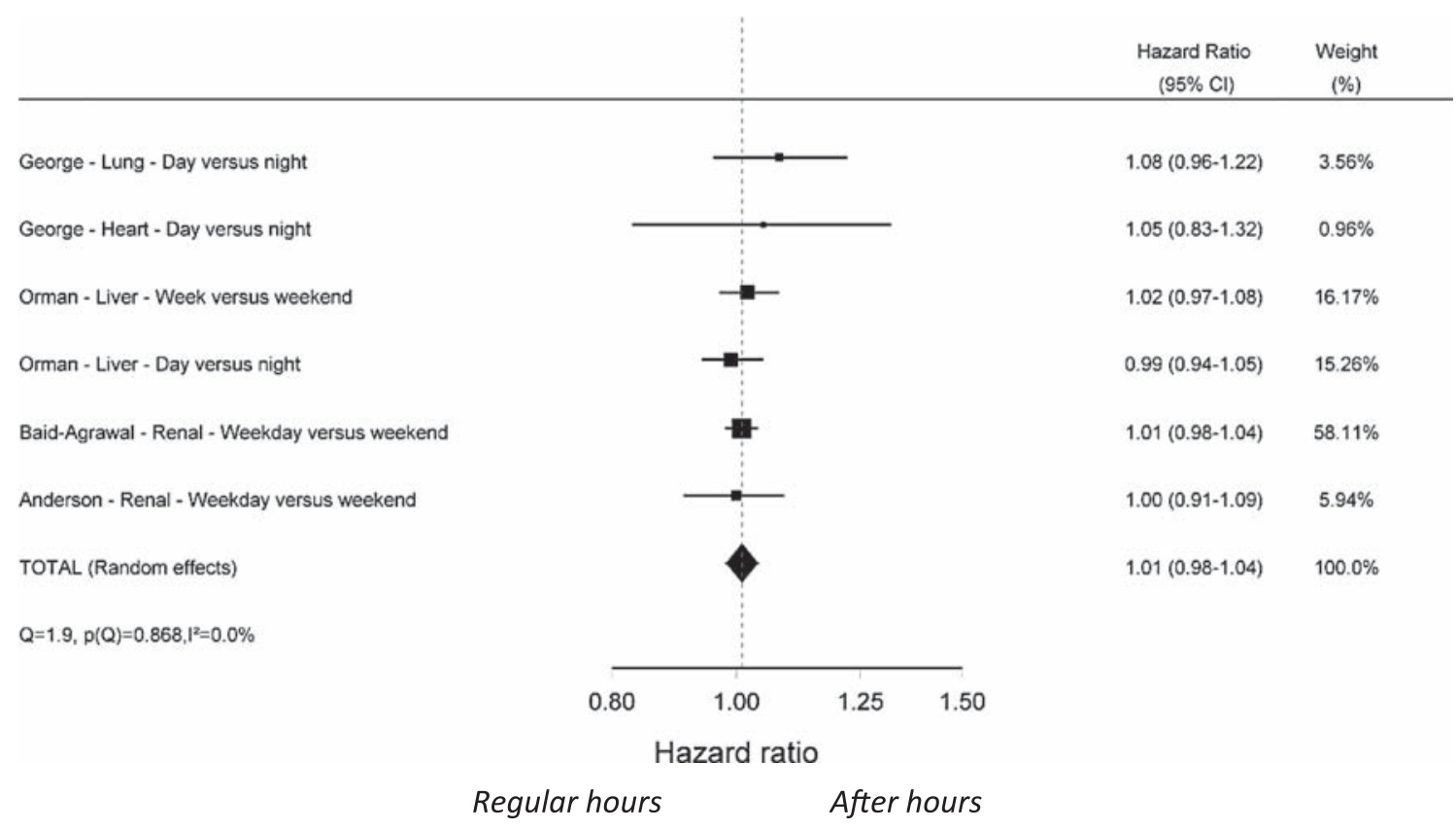

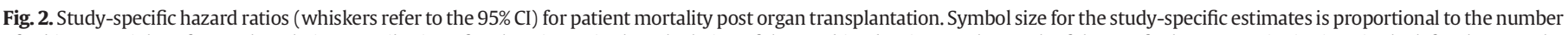

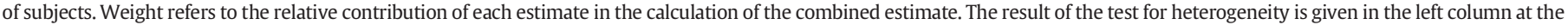
bottom of the figure. $\mathrm{CI}$ : confidence interval.

independently associated with a higher risk for in-hospital death, myocardial infarction and target vessel revascularization [adjusted odds ratio of 2.51 (p5\% CI, 1.39 to 4.56)] [31].

The negative effects of fatigue on technical and cognitive skills are well-documented both for surgeons and anesthesiologists [32-34]. However, these effects could not be linked to worse surgical outcomes in several studies [35,36]. In this regard, it is important to note that fatigue is only one factor jeopardizing surgical performance during after hours [30]. Worse postoperative outcomes for procedures starting outside the regular working hours have also been attributed to care transitions with consecutive information losses, lack of availability of specialists, less familiarity with the procedure, greater dependence on residents and unbefitting staffing [25]. Several subspecialties have specifically addressed these shortcomings by providing specialized care round the clock and hence guaranteeing the same standard of care and procedural outcome even during after hours [27]. This holds most likely also true for the majority of transplant centers, which could explain why the majority of the studies included in our review could not demonstrate a negative effect of performing transplant procedures after hours on outcome. In these centers, surgeons, anesthesiologists, nurses, perfusionists and the staff on the post-anesthesia care unit and the intensive care unit (ICU) are highly specialized for transplant procedures, familiar and experienced with this field of surgery. As a matter of fact, the experience of anesthesiologists with transplant surgery has recently been demonstrated to have a direct impact on mortality [37]. The availability of a dedicated team for transplant procedures is in contrast with other emergent surgery when subspecialized staff suddenly has to work with a broader variety of pathology. Besides that, residents and fellows are being trained preferentially during daytime while at night and during the weekends, the transplant surgery might be performed in first instance by the senior surgeon himself. This is however difficult to investigate since the level of experience of surgeons is rarely documented. Moreover, we have to be cautious to make this assumption since other studies hypothesized that elderly doctors are more likely to struggle with the impact of night-time work [38,39]. Further, it should be kept in mind that organ recipients are in close follow-up programs and went through extensive pre-operative investigations. Compared to the non-transplant population, the incidence of undiagnosed and untreated comorbidities affecting surgical outcomes is likely lower in transplant patients. Additionally, in transplant surgery the operative time is mainly determined by the availability of donor organs, timing of the procurement and CIT. The recipient's medical condition or an aggravation of symptoms as seen in common emergency cases does not play a roll. Normally, patients treated during after hours have higher acuity and greater urgency than patients treated during the day. Except for patients with acute organ failure undergoing highurgency transplantations, transplant surgery is performed with similar urgency at all times and patients have the equal acuity. In this regard, the setting of transplant surgery differs from acute critical illness because the patients undergoing transplantation are typically clinically stable. The lack of impact of starting after hours on outcomes could also be explained by the fact that drawbacks of after hours surgery might have been counterbalanced by a higher rate of ICU admissions. In fact, for non-transplant surgery, higher post-operative ICU admittance during nightly hours has been reported for Europe [25]. However, most organ transplant recipients, except kidney transplants, are postoperatively transferred to the ICU anyhow, regardless time of day. Other factors might even be in favor for surgery at night and during weekends like the more quiescent environment in the operation room, which has been proven to increase surgical performance and interruptions during surgery are correlated with more surgical errors [40]. Besides this, physiological changes observed at night such as decreased heart rate variability and a significant increase in pulse rate, suggest sympathetic dominance and possibly result in an increased alertness during the procedure [41].

The findings of this article must be considered in light of several important limitations. First, studies showed a great heterogeneity on different levels such as the definition of 'afterhours', the outcome measurements, the specific organ transplanted and published statistical data. The definitions of timing varied widely and while sleep deprivation and/or fatigue are important risk factors for operations performed at night, this does not necessarily hold true for surgeries performed at the weekend. The outcome measurements diverged widely among the studies. The most common published outcome measurements were patient survival, allograft failure, length of hospital stay and diverse complications. Seven studies researched patient survival; three studies 
after 30 days; two studies after 90 days; five studies after 1 year, three studies after 5 years. The allograft failure was investigated in six studies, the length of hospital stay was surveyed in 4 studies and complication rates were studied in five studies. The heterogeneity continues for the statistical data published in the articles. Hazard ratios were used in four studies, three studies used odds ratios and the remaining studies gave no ratios. That said, the results of the quantitative meta-analysis should be interpreted with caution. The meta-analysis analyzed reported hazard ratios that had been adjusted by the primary authors in multivariate analyses for confounders that were different between the individual studies. Moreover, the meta-analysis combines observations from different organs and different definitions of timing.

Second, the present review is unable to account for several confounders and immeasurable influences that might affect the acceptance of an offered graft. Unfortunately, most studies neither do report the recipient's medical condition nor disclose details on donor grafts. Of note, deceased-donor kidneys were recently found to be more frequently discarded during the weekends compared to weekday kidneys, even after adjusting for organ quality [42]. Various factors might attribute to this finding including reduced availability of manpower or resources as hemodialysis. In addition, both surgeons on call who have to cover various services and less experienced surgeons are more inclined to discard donor organs that other surgeons would accept [42]. Hence, depending on the surgeons' individual experience, the acceptance of organs may personally differ according to the time of day. Altogether, the populations compared in the present review (transplant surgery during after hours compared with to regular hours) might not necessarily match for all factors that are known to predict outcome, such as the donor risk, the recipient risk, primary transplantation vs. re-transplantation, and CIT. Third, the included studies failed to report the precise causes of recipients' death and/or complications, and information on important outcome parameters like incidence of re-transplantation was lacking.

Fourth, the relative contribution of anesthesia, surgery, patient-related factors, availability of medical resources, quality and intensity of postoperative care and other components to outcome remains largely unknown. It has to be acknowledged that the majority of outcome parameters are affected by far more factors than the effect of day time and fatigue on the performance of the transplant team, the dexterity of the surgeon and the alertness of the anesthesiologist. In particular long term outcomes are (also) influenced by a variety of known and unknown factors being effective in the postoperative trajectory. It is impossible to account for these factors as they are not reported in the individual studies.

Last, the authors used only used one data base to identify all potentially relevant observational retrospective cohort studies. This could cause a detection bias. In addition, only one author $(\mathrm{JH})$ performed data search and collection which might introduce a bias. However, the article selection was reviewed independently by a second author (SR).

In conclusion, the systematic review and meta-analysis evaluated the relationship between starting times in transplant surgery and postoperative outcome. The evidence was inconclusive for both postoperative mortality and morbidity. On the basis of the available evidence, it appears therefore impossible to give an unequivocal recommendation regarding starting times in transplant surgery. At current, it seems justified to determine timing primarily by the quest to minimize cold ischemia times, to optimize the preparation of the recipient and to enhance the availability of medical and technical resources. Further research is required to determine the optimal start time for transplant surgery, specifically addressing well-known confounders, optimizing data collection and using prospective study designs.

Supplementary data to this article can be found online at https://doi. org/10.1016/j.trre.2018.05.001.

\section{Authors' contributions}

Study design: SR.

Data collection and analysis: JH, SR.
Meta-analysis: SF.

Drafting article: JH, SR.

Critical revision of article: JP, DV.

Approval of article: JH.

\section{Conflict of interest}

The authors of this manuscript declare to have no conflicts of interest.

\section{References}

[1] Smilowitz NR, Gupta N, Ramakrishna H, Guo Y, Berger JS, Bangalore S. Perioperative major adverse cardiovascular and cerebrovascular events associated with noncardiac surgery. JAMA Cardiol 2017;2:181-7.

[2] Noordzij PG, Poldermans D, Schouten O, Bax JJ, Schreiner FAG, Boersma E. Postoperative mortality in The Netherlands: a population-based analysis of surgery-specific risk in adults. Anesthesiology 2010;112:1105-15.

[3] Gaba D, Howard S. Fatigue among clinicians and the safety of patients. N Engl J Med 2002;347:1249-55

[4] Landrigan C, Rothschild J, Cronin J, Kaushal R, Burdick E, Katz J, et al. Effect of reducing interns' work hours on serious medical errors in intensive care units. N Engl J Med 2004;351:1838-48.

[5] Kienzl-Wagner K, Schneiderbauer S, Bösmüller C, Schneeberger S, Pratschke J, Öllinger R. Nighttime procedures are not associated with adverse outcomes in kidney transplantation. Transpl Int 2013;26:879-85.

[6] Neuberger J. Day or night- does the time of liver transplantation matter? Nat Rev Gastroenterol Hepatol 2010;7:596-7.

[7] Debout A, Foucher Y, Trébern-Launay K, Legendre C, Kreis H, Mourad G, et al. Each additional hour of cold ischemia time significantly increases the risk of graft failure and mortality following renal transplantation. Kidney Int 2015;87:343-9.

[8] Moher D, Liberati A, Tetzlaff J, Altman D, Group TP. Preferred reporting items for systematic reviews and meta-analyses: the PRISMA statement. PLoS Med 2009; 6:e1000097.

[9] Charlson ME, Pompei P, Ales KL, MacKenzie CR. A new method of classifying prognostic comorbidity in longitudinal studies: development and validation. J Chronic Dis 1987; 40:373-83.

[10] Elixhauser A, Steiner C, Harris DR, Coffey RM. Comorbidity measures for use with administrative data. Med Care 1998;36:8-27.

[11] Kamath P, Wiesner RH, Malinchoc M, Kremers W, Therneau TM, Kosberg CL, et al. A model to predict survival in patients with end-stage liver disease. Hepatology 2001; 33:464-70.

[12] Hardy RJ, Thompson SGA. likelihood approach to meta-analysis with random effect Stat Med 1996;15:619-29.

[13] Higgins JP, Thompson SG. Quantigying heterogeneity in a meta-analysis. Stat Med 2002;21:1539-58.

[14] Anderson BM, Mytton JL, Evison F, Sharif A. Outcomes after weekend admission for deceased donor kidney transplantation: a population cohort study. Transplantation 2017; 101:2244-52.

[15] Baid-Agrawal S, Martus P, Feldman H, Kramer H. Weekend versus weekday transplant surgery and outcomes after kidney transplantation in the USA: a retrospective national database analysis. BMJ Open 2016;6:e010482.

[16] Manfredini R, Gallerani M, De Giorgi A, Boari B, Lamberti N, Manfredini F, et al. Lack of a "weekend effect" for renal transplant recipients : the hospital database of the emilia-romagna region of Italy. Angiology 2017;68:366-73.

[17] Fechner G, Pezold C, Hauser S, Gerhardt T, Müller SC. Kidney's nightshift, kidney's nightmare? Comparison of faylight and nighttime kidney transplantation: impact on complications and graft survival. Transplant Proc 2008;40:1341-4.

[18] Özdemir-van Brunschot DM, Hoitsma AJ, van der Jagt MF, D'Ancona FC, Donders RA, van Laarhoven CJ, et al. Nighttime kidney transplantation is associated with less pure technical graft failure. World J Urol 2016;34:955-61.

[19] Seow Y-Y, Alkari B, Dyer P, Riad H. Cold ischemia time, surgeon, time of day, and surgical complications. Transplantation 2004;77:1386-9.

[20] Shaw TM, Lonze BE, Feyssa EL, Segev DL, May N, Parsikia A, et al. Operative start times and complications after kidney transplantation. Clin Transpl 2012;26: E177-83.

[21] Orman ES, Hayashi PH, Dellon ES, Gerber DA, Barritt IVAS. Impact of nighttime and weekend liver transplants on graft and patient outcomes. Liver Transpl 2012;18: 558-65.

[22] Lonze BE, Parsikia A, Feyssa EL, Khanmoradi K, Araya VR, Zaki RF, et al. Operative start times and complications after liver transplantation. Am J Transplant 2010;10: 1842-9.

[23] George TJ, Arnaoutakis GJ, Merlo CA, Kemp CD, Baumgartner WA, Conte JV, et al. Association of operative time of day with outcomes after thoracic organ transplant. JAMA 2011:305:2193-9.

[24] Manfredini R, Gallerani M, De Giorgi A, Tiseo R, Lamberti N, Manfredini F, et al. Renal transplantation and outcome : does time of surgery matter? Prog Transplant 2016; 26:1-2.

[25] van Zaane B, van Klei WA, Buhre WF, Bauer P, Boerma EC, Hoeft A, et al. Nonelective surgery at night and in-hospital mortality. Eur J Anaesthesiol 2015 Jul;32:477-85.

[26] Sessler DI, Kurz A, Saager L, Dalton JE. Operation timing and 30-day mortality after elective general surgery. Anesth Analg 2011;113:1423-8. 
[27] Heller JA, Kothari R, Lin H-M, Levin MA, Weiner M. Surgery start time does not impact outcome in elective cardiac surgery. J Cardiothorac Vasc Anesth 2017;31:32-6.

[28] Kelz RR, Freeman KM, Hosokawa PW, Asch DA, Spitz FR, Moskowitz M, et al. Time of day is associated with postoperative morbidity: an analysis of the national surgical quality improvement program data. Ann Surg 2008;247:544-52.

[29] Whitlock EL, Feiner JR, Chen LL. Perioperative mortality, 2010 to 2014. Anesthesiology 2015;123:1312-21.

[30] Yount KW, Lau CL, Yarboro LT, Ghanta RK, Kron IL, Kern JA, et al. Late operating room start times impact mortality and cost for nonemergent cardiac surgery. Ann Thorac Surg 2015;100:1653-9.

[31] Glaser R, Naidu SS, Selzer F, Jacobs AK, Laskey WK, Srinivas VS, et al. Factors associated with poorer prognosis for patients undergoing primary percutaneous coronary intervention during off-hours. JACC Cardiovasc Interv 2008;1:681-8.

[32] Taffinder N, McManus I, Gul Y, Russell R, Darzi A. Effect of sleep deprivation on surgeons' dexterity on laparoscopy simulator. Lancet 1998;352:1191.

[33] Grantcharov TP, Bardram L, Peter F-J, Rosenberg J. Laparoscopic performance after one night on call in a surgical department: prospective study. BMJ 2001;323:1222-3.

[34] Wright MC, Phillips-Bute B, Mark JB, Stafford-Smith M, Grichnik KP, Andregg BC, et al. Time of day effects on the incidence of anesthetic adverse events. Qual Saf Health Care 2006;15:258-63.
[35] Vinden C, Nash DM, Rangrej J, Shariff SZ, Dixon SN, Jain AK, et al. Complications of daytime elective laparoscopic cholecystectomies performed by surgeons who operated the night before. JAMA 2013;310:1837-41.

[36] Chu MW, Stitt LW, Fox SA, Kiaii B, Quantz M, Guo L, et al. Prospective evaluation of consultant surgeon sleep deprivation and outcomes in more than 4000 consecutive cardiac surgical procedures. Arch Surg 2011;146:1080-5.

[37] Hofer I, Spivack J, Yaport M, Zerillo J, Reich DL, Wax D, et al. Association between anesthesiologist experience and mortality after orthotopic liver transplantation. Liver Transpl 2015;21:89-95.

[38] Katz JD. Issues of concern for the aging anesthesiologist. Anesth Analg 2001;92: 1487-92.

[39] Travis KW, Mihevc NT, Orkin FK, Zeitlin GL. Age and anesthetic practice: a regional perspective. J Clin Anesth 1999;11:175-86.

[40] Zheng B, Panton ONM, Al-Tayeb TA. Operative length independently affected by surgical team size: data from 2 Canadian hospitals. Can J Surg 2012;55:371-6.

[41] Amirian I, Toftegård Andersen L, Rosenberg J, Gögenur I. Decreased heart rate variability in surgeons during night shifts. Can J Surg 2014;57:300.

[42] Mohan S, Foley K, Chiles MC, Dube GK, Patzer RE, Pastan SO, et al. The weekend effect alters the procurement and discard rates of deceased donor kidneys in the United States. Kidney Int 2016;90:157-63. 